\title{
Análisis métrico de los blogs españoles de biblioteconomía y documentación (2006-2007)
}

\author{
Por Daniel Torres-Salinas, Álvaro Cabezas-Clavijo y Emilio Delgado-López-Cózar
}

\begin{abstract}
Resumen: Se analizan diversos indicadores de producción y participación en los blogs españoles de biblioteconomía y documentación. A partir de una muestra de 46 blogs de autoría personal y corporativa, se toman los 12 con mayor número de enlaces recibidos. Son los que obtienen más del $70 \%$ de los enlaces recogidos por el conjunto de blogs documentales. Se detecta una bajada productiva significativa a lo largo del período de estudio, manteniéndose constante el número de comentarios por entrada. Más del $50 \%$ de los comentarios son realizados por los propios autores de bitácoras documentales. Destaca un conjunto de 4 blogs, que pre-
\end{abstract} sentan indicadores por encima

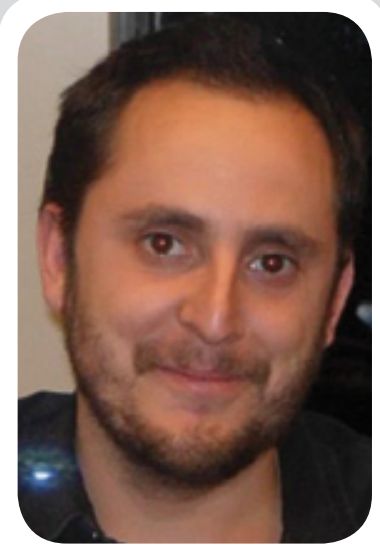

Daniel Torres Salinas es investigador del Centro de Investigación Médica Aplicada de la Universidad de Navarra y miembro del grupo EC3 (Evaluación de la Ciencia y de la Comunicación Científica) de la Universidad de Granada, donde recientemente ha leído su tesis doctoral.

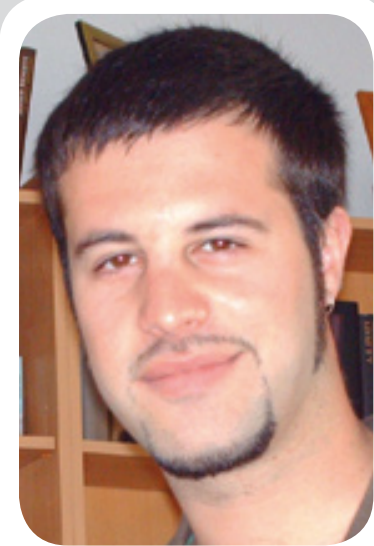

Álvaro Cabezas Clavijo es licenciado en documentación y miembro del grupo EC3 (Evaluación de la Ciencia y de la Comunicación Científica) de la Universidad de Granada. Su blog, Documentación, biblioteconomía e información es uno de los más visitados de la blogosfera documental hispana.

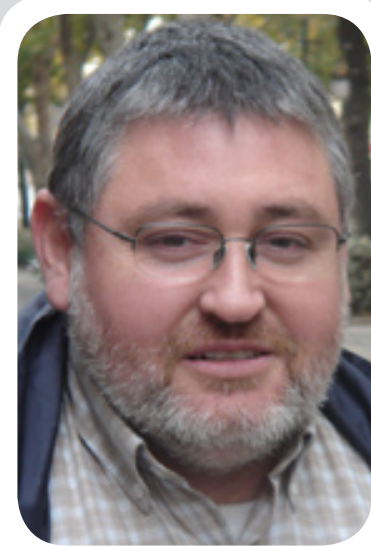

Emilio Delgado López-Cozar es profesor de metodología de la investigación en la Facultad de Biblioteconomía y Documentación y miembro del grupo EC3 (Evaluación de la Ciencia y de la Comunicación Científica) de la Universidad Granada. de la media en cuanto a producción y participación: Deakialli, Catorze, Documentalista enredado y Véase además.

Palabras clave: Blogs, Blogosfera, Blogmetría, Cibermetría, Biblioteconomía y documentación, España.

\section{Title: Metric analysis of Spanish library and information science blogs (2006-2007)}

Abstract: Several indicators of production and participation in Spanish library and information science blogs are analysed, based on a sample of 46 personal and corporate weblogs. The 12 blogs with the highest number of incoming links logged more than $70 \%$ of the total number of links received by the whole group. Our results detect an important decline in production during the study period, keeping constant the comments-per-post indicator. More than 50\% of comments are written by the library blog authors and 4 blogs show higher than average indicators of production and participation: Deakialli, Catorze, Documentalista enredado and Véase además.

Keywords: Blogs, Blogosphere, Blogmetrics, Cybermetrics, Library and information science, Spain.

Torres-Salinas, Daniel; Cabezas Clavijo, Álvaro; Delgado López-Cózar, Emilio. "Análisis métrico de los blogs españoles de Biblioteconomía y Documentación (2006-2007)”. En: El profesional de la información, 2008, enero-febrero, v. 17, n. 1, pp. 38-48.

DOI: $10.3145 /$ epi.2008.ene.04

\section{Introducción}

La popularización y el crecimiento de internet como medio de comunicación ha acabado derivando en los últimos años en la denominada web 2.0. Este nuevo ciberespacio se compone de un conjunto de herramientas complementarias entre sí (wikis, Flickr, Youtube, Panoramio, etc.) que facilitan la generación de contenidos en múltiples formatos por todo tipo de usuarios, y la interacción entre los mismos. Uno de los más genuinos y reconocidos es la blogosfera, formada por los weblogs, blogs o bitácoras. Estos sitios se caracterizan por presentar una ordenación cronológica inversa de sus contenidos, poseer una constante actualización y contar con frecuentes enlaces. Otros de sus aspectos fundamentales es la oportunidad que tienen los lectores de dejar comentarios en las diferentes entradas, lo que da lugar a fructíferos debates entre el autor y los lectores, por lo que se suele decir que "los blogs son la conversación" (Rojas, et al., 2005). 
En el ámbito bibliotecario y documental español el fenómeno blogger no ha pasado desapercibido y las bitácoras han estado presentes desde 2001 con la creación de Trucos de Google. El interés despertado en los círculos académicos ha quedado patente en diferentes acontecimientos acaecidos en los últimos meses, como el número especial que El profesional de la información (v. 16, n. 2, 2007) dedicó a dicho fenómeno o las recientes actividades paralelas que, con gran éxito de público, se llevaron a cabo en las últimas Jornadas españolas de documentación organizadas por Fesabid.

\section{http://www.fesabid.org/santiago2007/}

Buena prueba también es la variada y diversa bibliografía que se ha generado en torno al tema. A nivel general, distintos autores se han encargado de mostrar las utilidades de los weblogs para los documentalistas (Lledó, 2003; Sorli; Merlo, 2003; López; Martínez, 2005), su capacidad para la redifusión de contenidos mediante la sindicación (Franganillo; Catalán, 2005), caracterizado su terminología específica (Ferrada, 2006b) o señalado sus propias experiencias en la creación y gestión de las bitácoras (López; Penadés, 2007; Becerril, et al., 2007).

Específicamente también se ha estudiado la bibliogosfera (bibliogsfera o biblioblogosfera) es decir los weblogs especializados en biblioteconomía y documentación (ByD). Así, nos encontramos trabajos que han evaluado la calidad formal de 30 blogs académicos y de investigación (Jiménez; Salvador, 2007), caracterizado las redes sociales existentes entre la comunidad de blogs y algunos de sus indicadores cibermétricos (Flores; Legerén, 2005; Ros; Mateo, 2005a), realizado una historia de los blogs documentales hispanos (Ros; Mateo, 2005b) o lanzado propuestas de clasificación en función de su contenido (Leiva, 2006; Sedic, 2006).

Sin embargo, hasta el momento, no se ha realizado ningún acercamiento profundo a la blogosfera documental nacional desde una perspectiva puramente métrica, tal y como ha ocurrido en el mundo anglosajón (Bar-Ilan, 2005; Crawford, 2005), que nos permita describir nuestra comunidad. La única aportación que contamos a nivel nacional en este sentido fue el post ¿Se han estancado los blogs documentales? publicado en el blog Documentación, biblioteconomía e información el pasado mes de mayo (Cabezas, 2007).

Concretamente en esta entrada, a través del análisis de producción y participación en los dos primeros trimestres de 2006 y 2007, se evidenció un descenso en la producción y número de comentarios en seis blogs representativos del ámbito documental. Dicho post recibió más de 30 comentarios, en su mayoría de otros bloggers reconocidos, que valoraron positivamente el estudio, sugirieron ideas y líneas de investigación futuras, así como valiosas explicaciones del decaimiento en la producción y participación.

Teniendo en cuenta estos antecedentes, así como el debate y la expectación generada, parece interesante ampliar este breve estudio. El objetivo fundamental que se persigue es determinar si realmente se está produciendo un descenso en la actividad y si esta tendencia, además, es común a otros blogs de la disciplina. Asimismo introducimos nuevos indicadores que nos ayuden a describir otros aspectos como la longitud de las entradas, la frecuencia de publicación, los autores más célebres, los posts más comentados o el número de entradas que no han generado participación. En definitiva ofrecemos un análisis métrico, en este caso blogmétrico, que nos ayude a desvelar qué ha ocurrido en los últimos meses en la blogosfera documental española.

\section{Material y métodos}

Se realiza un estudio descriptivo longitudinal retrospectivo de 46 blogs personales y corporativos españoles especializados temáticamente en $\mathrm{ByD}$, por lo que la unidad de análisis se circunscribe, por tanto, a la blogosfera documental española. Sin embargo no es fácil delimitar esta comunidad con claridad debido al constate surgimiento de nuevos blogs y la desaparición de otros. Nos encontramos asimismo las propias dificultades conceptuales que comporta la delimitación de un campo multidisciplinar como éste (Jiménez, et al., 2006). Según recientes estimaciones, existen unas 80 bitácoras españolas relativas a $\mathrm{ByD}$, desde bibliotecas públicas, universitarias y especializadas a personales o de asociaciones e instituciones (Sedic, 2006).

Partiendo de este grupo, para el presente trabajo se han considerado y delimitado como unidad de análisis todos los que aparecen recopilados en el esquema clasificatorio propuesto por el equipo del Sedic blog (2006) bajo los encabezamientos "Blogs colectivos de asociaciones o instituciones", "Blogs personales" y "Blogs individuales que aportan contenidos muy personales, de carácter heterogéneo" (un total de 46 sitios, como se dijo). Esta clasificación es una de las más recientes y exhaustivas, y una de las aproximaciones más coherentes a una categorización de los blogs de ByD. El marco cronológico corresponde a los meses comprendidos entre enero de 2006 y abril de 2007, abarcándose por tanto un total de 485 días.

Una vez establecida y delimitada la unidad de análisis se ha calculado para cada uno de los 46 blogs su "Autoridad" (Technorati authority) un indicador creado y proporcionado por el buscador especializado Technorati, que se entiende como el número de blogs citantes en los últimos seis meses (Carroll, 2007). Esta medida posee algunas limitaciones, como que no distingue los 
enlaces enviados desde el blogroll; no contempla los links recibidos desde sitios web que no sean blogs; y depende de la fecha en la que se realizó la búsqueda. Sin embargo nos ofrece una aproximación válida del impacto de un blog dentro de su propia comunidad, y del reconocimiento que otros autores le otorgan. Una vez calculada la "Autoridad", se han seleccionado los situados en el primer cuartil del ranking a fin de calcular para cada uno de ellos los indicadores de la tabla 1.

http://technorati.com/

\begin{tabular}{|l|c|}
\hline Indicador & Abrev. \\
\hline Número de posts o entradas & P \\
\hline Número de posts por día & PxD \\
\hline Número de palabras contenidas en los posts & PR \\
\hline Número de palabras por post & PRXD \\
\hline Número de comentarios recibidos & $\mathbf{C}$ \\
\hline Número de comentarios por post & CxP \\
\hline Número de posts sin comentarios & PSC \\
\hline
\end{tabular}

Tabla 1. Indicadores empleados en el análisis de los blogs españoles de ByD

Asimismo se ha realizado una caracterización específica de los comentarios clasificándolos en tres tipos diferentes: sitio.

- Auto: los realizados por un autor en su propio

- Internos: comentario recibido por autores de alguno de los 46 blogs de ByD pertenecientes a la unidad de análisis.

- Externos: cuando el comentario no proviene de ninguno de los dos anteriores.

\section{Resultados y discusión}

\section{Resultados generales}

La mayor parte de los blogs presentan algunas características comunes que le otorgan al grupo cierta homogeneidad (tabla 2). En su mayor parte son personales $(90 \%)$ por lo que no se adscriben a asociaciones o instituciones; suelen ser redactados por una persona encargada tanto de su gestión como del mantenimiento de los contenidos (76\%); son escritos en castellano $(91 \%)$ y su fecha de inicio suele situarse entre 2004$2006(80 \%)$.

Centrando la atención en el indicador de "Autoridad" se constata como 12 blogs acumulan más del $70 \%$ de los enlaces. Los más enlazados son en su mayoría de tipo personal y gestionados por uno o dos autores.
Diez de ellos se redactan en castellano, mientras que los dos restantes usan el catalán como lengua principal. La mayor parte de estos 12 blogs comenzaron su andadura entre 2003 y 2004 y son los que seleccionamos finalmente para el cálculo de los indicadores propuesto en la tabla 2.

El número de posts producidos por los 12 blogs más enlazados ha sido de 1.970 lo que supone una media de 123 entradas al mes (tabla 3). Comparados el primer cuatrimestre de 2006 y 2007, existe una diferencia significativa ya que en los cuatros primeros meses de 2006 el número de posts fue de 610 mientras que en el mismo marco, para 2007, esta cifra sólo alcanza 418. Este descenso se refleja asimismo en el número de posts por día. El valor general para PxD es de 4, siendo los meses de enero y febrero de 2006 los únicos en los que se alcanzaron 5 o más diarios, estas cantidades ya no se vuelven a alcanzar y en el último mes, abril de 2007, se llegó al mínimo con 2,8 entradas diarias.

\section{"La mayor parte de los blogs son personales $(90 \%)$ y su fecha de inicio suele situarse entre 2004-2006 (80\%)"}

En cuanto a la longitud de los posts (número de palabras), el conjunto de blogs ha escrito casi medio millón de palabras. Este indicador muestra un carácter decreciente, como es lógico: a un menor número de posts le equivale una menor cantidad de texto. La longitud estándar de los posts se situó en 253 palabras, valor que se mantiene bastante constante a lo largo del período, lo que revela que aunque se escriban menos posts suelen ser casi siempre del mismo tamaño.

Otro de los aspectos interesantes son los comentarios, denotativos, en principio, de la capacidad de un autor para generar debate en la blogosfera así como de la actividad de sus lectores. El número de ellos en total fue de 5.729, con tendencia a disminuir, ya que de los más de 2.000 que se realizaron en los cuatro primeros meses de 2006 se ha pasado en 2007 a no superar los 1.500. Sin embargo, este descenso va unido de nuevo al número de posts redactados. Si tomamos el indicador CxP vemos que este valor se sitúa en 2,9 y a lo largo del período no se identifica ninguna tendencia. Otro de los aspectos destacados es el número de posts que no han recibido ningún comentario (PSC), que en total son $822(42 \%)$. Este valor es bastante estable, y tan sólo en enero y febrero de 2006 se mostraron porcentajes muy por debajo de la media. 


\begin{tabular}{|c|c|c|c|c|c|c|c|c|c|}
\hline Rank & NOMBRE & URL & $\begin{array}{l}\text { Auto- } \\
\text { ridad }\end{array}$ & $\begin{array}{c}\% \\
\begin{array}{c}\text { Autori- } \\
\text { dad }\end{array}\end{array}$ & $\begin{array}{c}\% \\
\text { Acumu- } \\
\text { lado }\end{array}$ & $\begin{array}{l}\text { Tipolo- } \\
\text { gía }\end{array}$ & $\begin{array}{c}\text { No } \\
\text { Auto- } \\
\text { res }\end{array}$ & Lengua & $\begin{array}{l}\text { Año } \\
\text { de } \\
\text { Inicio }\end{array}$ \\
\hline 1 & Catorze.blog & http://www.catorze.com/blog & 164 & 13,19 & 13,19 & Personal & 1 & Castellano & 2003 \\
\hline 2 & $\begin{array}{l}\text { Deakialli docu- } \\
\text { Mental }\end{array}$ & http://www.deakialli.com/ & 150 & 12,07 & 25,26 & Personal & 2 & Castellano & 2003 \\
\hline 3 & $\begin{array}{l}\text { El documentalista } \\
\text { enredado }\end{array}$ & http://www.documentalistaenredado.net/ & 138 & 11,10 & 36,36 & Personal & 2 & Castellano & 2004 \\
\hline 4 & $\begin{array}{l}\text { Úsalo. Usabilidad } \\
\text { para todos }\end{array}$ & http://usalo.es/ & 95 & 7,64 & 44,00 & Personal & 2 & Castellano & 2004 \\
\hline 5 & Véase además & http://www.veaseademas.com & 84 & 6,76 & 50,76 & Personal & 4 & Castellano & $2004^{(=)}$ \\
\hline 6 & \begin{tabular}{|l} 
Documentación, \\
biblioteconomía e \\
información
\end{tabular} & http://www.lacoctelera.com/documentacion & 48 & 3,86 & 54,62 & Personal & 1 & Castellano & 2005 \\
\hline 7 & $\begin{array}{l}\text { Recuperación de } \\
\text { información en la } \\
\text { web }\end{array}$ & http://irsweb.blogspot.com/ & 48 & 3,86 & 58,48 & Personal & 1 & Castellano & 2004 \\
\hline 8 & Sedic blog & http://blog.sedic.es & 35 & 2,82 & 61,30 & \begin{tabular}{|c|} 
Asoc. E \\
Inst.
\end{tabular} & $6^{(+)}$ & Castellano & 2006 \\
\hline 9 & $\begin{array}{l}\text { Gamoia. bloc } \\
\text { d'una bibliotecària }\end{array}$ & http://gamoia.bloc.cat/ & 33 & 2,65 & 63,95 & Personal & 1 & Catalán & 2005 \\
\hline 10 & Bibliometría & http://www.bibliometria.com/ & 32 & 2,57 & 66,53 & Personal & $1^{(+)}$ & Castellano & $2005^{(=)}$ \\
\hline 11 & Bauen blog & http://bauenblog.info/ & 30* & 2,41 & 68,94 & Personal & 2 & Catalán & 2004 \\
\hline 12 & Métodos de busca & http://www.metodosdebusca.com & 30 & 2,41 & 71,36 & Personal & 1 & Castellano & 2001 \\
\hline 13 & $\begin{array}{l}\text { La imagen social } \\
\text { del bibliotecario }\end{array}$ & http://lisdb.blogspot.com/ & 25 & 2,01 & 73,37 & Personal & 1 & Castellano & 2006 \\
\hline 14 & Alas y balas & http://www.alasybalas.com/ & 24 & 1,93 & 75,30 & Personal & 1 & Castellano & 2003 \\
\hline 15 & $\begin{array}{l}\text { Biblioblog. bitácora } \\
\text { informativa sobre } \\
\text { bibliotecas }\end{array}$ & $\begin{array}{l}\text { http://www.interreg-eet.info/weblogs/biblio- } \\
\text { blog/index.html }\end{array}$ & 23 & 1,85 & 77,15 & $\begin{array}{l}\text { Asoc. E } \\
\text { Inst. }\end{array}$ & 6 & Castellano & 2003 \\
\hline 16 & $\begin{array}{l}\text { Bibliotecosas: silva } \\
\text { bibliotecaria de } \\
\text { varia lección: } \\
\end{array}$ & http://bibliotecosas.blogia.com/ & 23 & 1,85 & 79,00 & Personal & 1 & Castellano & 2004 \\
\hline 17 & Anécdota? & http://www.anecdotica.net/ & 21 & 1,69 & 80,69 & Personal & 1 & Castellano & 2005 \\
\hline 18 & Open Access & http://weblogs.madrimasd.org/openaccess/ & 19 & 1,53 & 82,22 & Personal & 1 & Castellano & 2006 \\
\hline 19 & $\begin{array}{l}\text { @rchivista: una } \\
\text { mirada al mundo } \\
\text { de los archivos } \\
\end{array}$ & http://archivista.wordpress.com/ & 17 & 1,37 & 83,58 & Personal & 1 & Castellano & 2006 \\
\hline 20 & A propósito de & http://www_apropositode.blogspot.com & 17 & 1,37 & 84,95 & Personal & 1 & Castellano & 2005 \\
\hline 21 & TekaTeka & http://tekateka.blogspot.com/ & 16 & 1,29 & 86,24 & Personal & 1 & Castellano & 2004 \\
\hline 22 & Archivistica.net & http://www.archivistica.blogspot.com & 15 & 1,21 & 87,45 & Personal & 1 & Castellano & 2003 \\
\hline 23 & Interneteando & $\begin{array}{l}\text { http://interneteando-lara.blogspot.com/index. } \\
\text { html }\end{array}$ & 15 & 1,21 & 88,65 & Personal & 1 & Castellano & 2006 \\
\hline 24 & $\begin{array}{l}\text { El documentalista } \\
\text { audaz }\end{array}$ & http://sirmijail.blogspot.com/ & 14 & 1,13 & 89,78 & Personal & 1 & Castellano & 2005 \\
\hline 25 & Geekteca & http://geekteca.blogspot.com/ & 13 & 1,05 & 90,82 & Personal & 1 & Castellano & 2004 \\
\hline 26 & $\begin{array}{l}\text { biBLOGteca. bBlog } \\
\text { de Asnabi }\end{array}$ & http://asnabi.blogspirit.com & 12 & 0,97 & 91,79 & \begin{tabular}{|c|}
$\begin{array}{c}\text { Asoc. E } \\
\text { Inst. }\end{array}$ \\
\end{tabular} & $\begin{array}{r}\text { Sin } \\
\text { dato }\end{array}$ & Castellano & 2006 \\
\hline 27 & $\begin{array}{l}\text { Outbook: diseño } \\
\text { web, usabilidad y } \\
\text { documentación }\end{array}$ & http://outbook.blogspot.com/ & 11 & 0,88 & 92,68 & Personal & 1 & Castellano & 2004 \\
\hline 28 & $\begin{array}{l}\text { Expediente, web, } \\
\text { blog y otros } \\
\text { documentos del } \\
\text { montón }\end{array}$ & http://documentalqueridowatson.blogspot.com & $10 *$ & 0,80 & 93,48 & Personal & 1 & Castellano & 2005 \\
\hline 29 & Servicio de alerta & http://docdigital.typepad.com/ & 10 & 0,80 & 94,28 & Personal & 3 & Castellano & 2004 \\
\hline
\end{tabular}

Tabla 2. Características de los blogs españoles de ByD con mención del indicador de "Autoridad" elaborado por Technorati

- Búsqueda en Technorati realizada el 18 de mayo de $2007 . \quad$ _ _ _ Cambio url.

- En gris: blogs seleccionados para el cálculo de los indicadores _ ${ }^{* * *}$ No actualizado en 2007.

propuestos en la tabla 1.

- *álculo manual.

- ${ }^{(+)}$Colaboraciones frecuentes de otros autores.

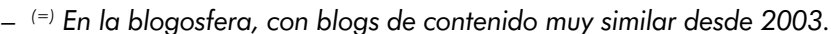




\begin{tabular}{|c|c|c|c|c|c|c|c|c|c|}
\hline Rank & NOMBRE & URL & $\begin{array}{l}\text { Auto- } \\
\text { ridad }\end{array}$ & $\begin{array}{c}\% \% \\
\text { Autori- } \\
\text { dad } \\
\end{array}$ & \begin{tabular}{|c|}
$\%$ \\
Acumu- \\
lado \\
\end{tabular} & $\begin{array}{l}\text { Tipolo- } \\
\text { gía }\end{array}$ & \begin{tabular}{|c|} 
No \\
Auto- \\
res \\
\end{tabular} & Lengua & $\begin{array}{c}\text { Año } \\
\text { de } \\
\text { Inicio }\end{array}$ \\
\hline 30 & $\begin{array}{l}\text { Ranganatha2: } \\
\text { atrévete a pensar } \\
\text { (Think ahead) }\end{array}$ & http://www.espacioblog.com/ranganatha2 & 10 & 0,80 & 95,09 & Personal & 2 & Castellano & 2006 \\
\hline 31 & Tentándole & http://tentandole.blogsome.com/ & 10 & 0,80 & 95,89 & Personal & 1 & Castellano & 2006 \\
\hline 32 & Baiget info notas & http://baiget.blogspot.com/ & 9 & 0,72 & 96,62 & Personal & 1 & Castellano & 2004 \\
\hline 33 & EC3noticias & http://ec3noticias.blogspot.com/ & 7 & 0,56 & 97,18 & \begin{tabular}{|l|} 
Asoc. $\mathrm{E}$ \\
Inst.
\end{tabular} & $1^{(+)}$ & Castellano & 2005 \\
\hline 34 & $\begin{array}{l}\text { Bibliotecaescolar. } \\
\text { net }\end{array}$ & http://antoniotejero.blogspot.com/ & 7 & 0,56 & 97,74 & Personal & 1 & Castellano & 2005 \\
\hline 35 & Mixobitácora & http://mixobitacora.blogspot.com/ & 5 & 0,40 & 98,15 & Personal & 1 & Castellano & 2005 \\
\hline 36 & $\begin{array}{l}\text { Vida de una men- } \\
\text { ganita }\end{array}$ & http://menganita.blogspot.com/ & 5 & 0,40 & 98,55 & Personal & 1 & Castellano & $\begin{array}{l}\text { Sin } \\
\text { dato }\end{array}$ \\
\hline 37 & $\begin{array}{l}\text { Bibliotecas digi- } \\
\text { tales* }\end{array}$ & http://bibliotecas-digitales.blogspot.com/ & 5 & 0,40 & 98,95 & Personal & 1 & Castellano & 2006 \\
\hline 38 & Biblio's & http://libiblios.blogspot.com/ & 4 & 0,32 & 99,27 & Personal & 1 & Catalán & 2004 \\
\hline 39 & Documentamanía & http://documentamania.blogspot.com/ & 3 & 0,24 & 99,51 & Personal & 1 & Castellano & 2006 \\
\hline 40 & $\begin{array}{l}\text { Archiveros } \\
\text { Españoles en la } \\
\text { Función Pública }\end{array}$ & http://archiverosefp.blogspot.com/ & 2 & 0,16 & 99,67 & $\begin{array}{l}\text { Asoc. } \mathrm{E} \\
\text { Inst }\end{array}$ & $\begin{array}{l}\text { Sin } \\
\text { dato }\end{array}$ & Castellano & 2006 \\
\hline 41 & Bibliosalud & http://www. lacoctelera.com/bibliosalud & 2 & 0,16 & 99,84 & Personal & 1 & Castellano & 2006 \\
\hline 42 & $\begin{array}{l}\text { Joana Albret } \\
\text { bibliotekonomia } \\
\text { mintegia }\end{array}$ & http://www.eibar.org/blogak/joana-albret & 1 & 0,08 & 99,92 & Personal & 1 & Euskera & 2007 \\
\hline 43 & La mirilla digital & http://mirilladigital.blogspot.com/ & 1 & 0,08 & 100,00 & Personal & 1 & Castellano & 2006 \\
\hline 44 & De todo un poko & http://dtnpk.blogspot.com & 0 & 0,00 & 100,00 & Personal & 1 & Castellano & 2005 \\
\hline 45 & $\begin{array}{l}\text { Alfin: avances de } \\
\text { la alfabetización } \\
\text { Informacional }\end{array}$ & http://alfin.blogspirit.com & $\mathbf{X}^{* *}$ & 0,00 & 100,00 & Personal & 1 & Castellano & 2005 \\
\hline 46 & $\begin{array}{l}\text { El hombre irrazo- } \\
\text { nable }\end{array}$ & http://victormacias.blogia.com/ & $\mathbf{X}^{* * *}$ & 0,00 & 100,00 & Personal & 1 & Castellano & 2006 \\
\hline
\end{tabular}

Tabla 2 (continuación). Características de los blogs españoles de ByD con mención del indicador de "Autoridad" elaborado por Technorati

- Búsqueda en Technorati realizada el 18 de mayo de 2007.

- En gris: blogs seleccionados para el cálculo de los indicadores _ *** No actualizado en 2007.

propuestos en la tabla 1.

* Cálculo manual.

- ${ }^{+}$Colaboraciones frecuentes de otros autores.

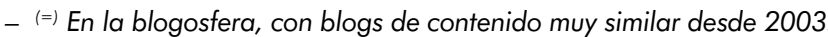

\begin{tabular}{|l|c|c|c|c|c|c|c|c|}
\hline \multicolumn{1}{|c|}{ Mes } & P & PXD & PR & PRxP & C & CxP & PSC & \%PSC \\
\hline 1. Enero & 190 & 6,1 & 47.501 & 250 & 658 & 3,5 & 65 & 34 \\
\hline 2. Febrero & 141 & 5,0 & 37.887 & 269 & 698 & 5,0 & 35 & 25 \\
\hline 3. Marzo & 140 & 4,5 & 34.849 & 249 & 365 & 2,6 & 57 & 41 \\
\hline 4. Abril & 139 & 4,6 & 31.357 & 226 & 370 & 2,7 & 54 & 39 \\
\hline 5. Mayo & 126 & 4,1 & 28.758 & 228 & 235 & 1,9 & 63 & 50 \\
\hline 6. Junio & 117 & 3,9 & 34.321 & 293 & 330 & 2,8 & 53 & 45 \\
\hline 7. Julio & 96 & 3,1 & 24.858 & 259 & 261 & 2,7 & 42 & 44 \\
\hline 8. Agosto & 83 & 2,7 & 24.357 & 293 & 309 & 3,7 & 31 & 37 \\
\hline 9. Septiembre & 109 & 3,6 & 24.633 & 226 & 171 & 1,6 & 60 & 55 \\
\hline 10. Octubre & 136 & 4,4 & 42.937 & 316 & 302 & 2,2 & 55 & 40 \\
\hline 11. Noviembre & 143 & 4,8 & 37.551 & 263 & 349 & 2,4 & 62 & 43 \\
\hline 12. Diciembre & 132 & 4,3 & 28.484 & 216 & 252 & 1,9 & 52 & 39 \\
\hline 13. Enero & 121 & 3,9 & 29.371 & 243 & 316 & 2,6 & 57 & 47 \\
\hline 14. Febrero & 117 & 4,2 & 27.584 & 236 & 267 & 2,3 & 49 & 42 \\
\hline 15. Marzo & 96 & 3,1 & 21.854 & 228 & 599 & 6,2 & 51 & 53 \\
\hline 16. Abril & 84 & 2,8 & 22.034 & 262 & 247 & 2,9 & 36 & 43 \\
\hline Total general & $\mathbf{1 . 9 7 0}$ & $\mathbf{4 , 1}$ & $\mathbf{4 9 8 . 3 3 6}$ & $\mathbf{2 5 3}$ & $\mathbf{5 . 7 2 9}$ & $\mathbf{2 , 9}$ & $\mathbf{8 2 2}$ & $\mathbf{4 2}$ \\
\hline
\end{tabular}

Tabla 3. Indicadores generales para los blogs de ByD: posts, palabras y comentarios 
En la tabla 4 se han identificado y caracterizado temáticamente los posts que han recibido 17 o más comentarios, destacando el Criterio Hotmail, de Catorze, con 337. Sin embargo esta tabla ha de leerse con ciertas reservas. Si tomamos como botón de muestra los tres casos más comentados, se observa la escasa relación que guardan con la documentación; dos de ellos son de tipo generalista (Criterio Hotmail y ¿Qué es la ciencia?) y otro (Marina d'Or apesta) es una breve frase al margen de la documentación. Los usuarios de los blogs que han dejado los comentarios son personas alejadas, en su gran mayoría, de la disciplina y el listado completo dista mucho de un debate serio y fructífero.

No siempre, por tanto, la cantidad de comentarios es una medida de la influencia en el campo; normal-
"La cantidad de comentarios no siempre es una medida de la influencia en el campo; normalmente los posts desenfadados sobre temas poco especializados son los que más reacciones desencadenan"

mente los posts con temas poco especializados, generalistas y de carácter desenfadado son los que más reacciones desencadenan. Si atendemos a toda la tabla, observamos que tan sólo un tercio pueden ser considerados de temática profesional. Otro tanto corresponde a

\begin{tabular}{|c|c|c|c|c|c|c|}
\hline Rank & Blog & Título del post & C & Año & Mes & Tema \\
\hline 1 & Catorze & El criterio Hotmail & 337 & 2007 & Marzo & No ByD \\
\hline 2 & Véase & Marina d'Or apesta & 88 & 2006 & Febrero & No ByD \\
\hline 3 & Bibliometria & ¿Qué es ciencia? & 85 & 2006 & Agosto & Profesional \\
\hline 4 & Deakialli & $\begin{array}{l}4 \text { velas para Deakialli, cuatro años } \\
\text { blogueando }\end{array}$ & 38 & 2007 & Marzo & Personal \\
\hline 5 & Catorze & Google Co-op y los blogs sobre ByD & 30 & 2006 & Octubre & Blogs \\
\hline 6 & Catorze & Hotmail & 30 & 2007 & Abril & No ByD \\
\hline 7 & Deakialli & Tres años de Deakialli & 28 & 2006 & Marzo & Personal \\
\hline 8 & Deakialli & Se pide opinión. La utilidad de los blogs & 27 & 2006 & Julio & Blogs \\
\hline 9 & Bibliometria & Una cronología del fraude científico & 26 & 2006 & Febrero & Profesional \\
\hline 10 & Catorze & Repositorio de comentarios & 25 & 2007 & Marzo & No ByD \\
\hline 11 & Deakialli & Deakialli.com & 24 & 2006 & Noviembre & Personal \\
\hline 12 & Véase & $\begin{array}{l}\text { Tips para que tu trabajo no interfiera } \\
\text { en tu bitácora }\end{array}$ & 24 & 2006 & Febrero & Blogs \\
\hline 13 & Véase & Wikis como organizadores personales & 24 & 2006 & Febrero & Profesional \\
\hline 14 & Catorze & Pasado, presente, futuro & 24 & 2006 & Agosto & Personal \\
\hline 15 & Úsalo & Fraudes medallistas & 23 & 2006 & Abril & Profesional \\
\hline 16 & $\begin{array}{l}\text { Documentalista } \\
\text { Enredado }\end{array}$ & Carpe diem bloguer, carpe diem & 20 & 2006 & Enero & Blogs \\
\hline 17 & Deakialli & Deakialli en Localia TV & 20 & 2006 & Junio & Personal \\
\hline 18 & Documentacion & Historias de terror (II) & 20 & 2006 & Junio & Profesional \\
\hline 19 & Véase & $\begin{array}{l}\text { Exhaustividad y precisión en SRIs basa- } \\
\text { dos en tagging }\end{array}$ & 20 & 2006 & Julio & Profesional \\
\hline 20 & Catorze & ISSN para weblogs: intento fallido & 19 & 2006 & Enero & Blogs \\
\hline 21 & Documentacion & Fallo de seguridad en El país? & 18 & 2006 & Enero & Profesional \\
\hline 22 & Úsalo & Usabilidad para principiantes & 18 & 2006 & Enero & Profesional \\
\hline 23 & Deakialli & $\begin{array}{l}\text { Los estudios universitarios de bibliote- } \\
\text { conomía y documentación existen ¿lo } \\
\text { sabías? }\end{array}$ & 18 & 2007 & Marzo & Profesional \\
\hline 24 & Deakialli & $\begin{array}{l}\text { Blog da asesoría de bibliotecas escola- } \\
\text { res }\end{array}$ & 18 & 2007 & Abril & Blogs \\
\hline
\end{tabular}

Tabla 4. Ranking de posts con mayor número de comentarios en los blogs de ByD: número de comentarios y tema 
cuestiones sin relación alguna con la documentación o relativas a celebraciones y anuncios que el autor realiza sobre su bitácora. El tercio restante se relaciona directamente con diversos aspectos del universo blogger.

En la figura 1 se presenta una caracterización de los 5.729 comentarios recopilados según el origen del comentarista. Predominan con un $43 \%$ los realizados por lectores ajenos a los blogs de ByD, mientras que un $27 \%$ fueron internos. El 30\% de autocomentarios indica el interés que los autores se toman no sólo en escribir sus entradas sino también en responder a los lectores. Si tomamos conjuntamente los autocomentarios y los internos se obtiene un $57 \%$, cifra bastante elevada.

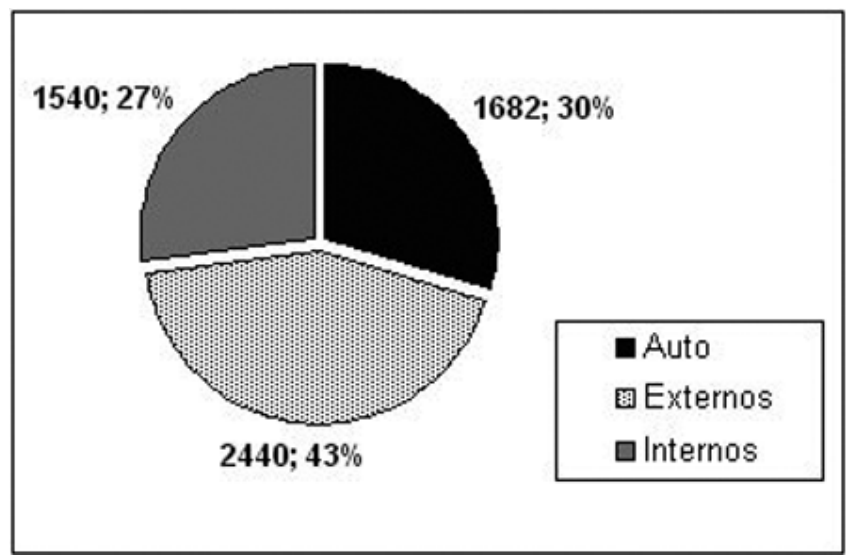

Figura 1. Porcentaje y número de comentarios según su origen (auto, externos e internos) para los blogs de ByD

\section{Resultados por blog}

En la tabla 5 se describen más detalladamente los doce blogs más enlazados presentándose los autores, sus profesiones y otros medios de comunicación empleados por ellos en la difusión de sus ideas. Como se observa, prácticamente todos los bloggers están vinculados a alguna profesión relacionada directamente con la ByD y más de la mitad pertenecen a Exit (Directorio de expertos en el tratamiento de la información). La mayor parte de ellos, además, ha empleado en alguna ocasión otros medios de comunicación al margen de su bitácora, como es el caso las revistas, preferentemente científicas y nacionales, o las comunicaciones a congresos.

\section{http://www.directorioexit.info/}

En la tabla 6 se muestran los indicadores para cada uno de los blogs. Si atendemos a la actividad produc-

\footnotetext{
"Todos los bloggers están vinculados a la ByD y más de la mitad pertenecen a Exit"
}

tiva, el más destacado ha sido Deakialli con 342 entradas seguido de Véase además con 260. Ambos son redactados por más de una persona. Los menos actualizados durante el período han sido Métodos de busca y Úsalo. Ninguno supera durante el período el post por día publicado, siendo la media de publicación de una entrada cada tres días $(0,34)$. Entre los que publican las más extensas, sin entrar en su calidad, se encuentra el Documentalista enredado con una extensión de 602 palabras de media. El blog con una ratio más baja ha sido Véase además con 98. La longitud media de los posts se mueve en un arco bastante amplio por lo que no existe homogeneidad para este indicador.

En cuanto a los comentarios, el más destacado, para el indicador $C$, ha sido Catorze con 1.133, seguido de Deakialli con 1.107. Además, tal y como se reflejó en la tabla 4 son los que tienen mayor capacidad para generar posts con un gran número de comentarios. Respecto a la cantidad de comentarios por post, sólo dos blogs alcanzan o superan los 5 CxP: Catorze y Documentación, biblioteconomía e información. La zona baja vuelve a ocuparla Métodos de busca, que prácticamente no ha recibido ninguno. Igualmente hay que señalar la importante brecha existente entre los cuatro blogs que menos comentarios reciben (Métodos de busca, Bauen, RI en la web y Sedic) y los ocho restantes, que alcanzan cifras de participación muy estimables. Estas diferencias también quedan reflejadas para el indicador número de posts sin comentarios, pues sólo tres blogs consiguen que menos del $20 \%$ de sus entradas se queden sin comentarios (Úsalo; Documentación, Biblioteconomía e información; y el Documentalista enredado).

En la figura 2 resumimos cómo se sitúan los blogs en relación con la media general de dos indicadores importantes: Número de posts y Número de comentarios por post. Son cuatro blogs los que dominan la escena, correspondientes a los que ocupan el margen superior

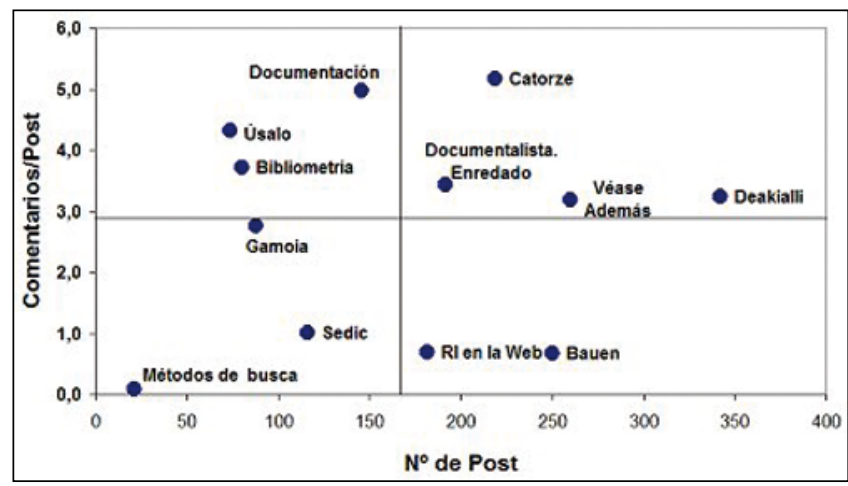

Figura 2. Posición de los blogs de ByD respecto a la media general de los indicadores "Número de posts" (P) y "Número de comentarios por post" (CxP)

*Línea horizontal paralela a abscisas: media general de Comentarios por post $=2,9$

*Línea vertical paralela a ordenadas: media general de Número de posts $=164$ 


\begin{tabular}{|c|c|c|c|c|c|c|c|c|c|}
\hline Autor & Blog & Profesión* & $\begin{array}{c}\text { Ficha } \\
\text { Exit }\end{array}$ & Libros & $\begin{array}{c}\text { Revis- } \\
\text { tas }\end{array}$ & $\begin{array}{l}\text { Otros } \\
\text { blogs }\end{array}$ & $\begin{array}{c}\text { Otros } \\
\text { me- } \\
\text { dios }\end{array}$ & $\begin{array}{l}\text { Con- } \\
\text { gre- } \\
\text { sos }\end{array}$ & $\begin{array}{l}\text { Cur- } \\
\text { sos }\end{array}$ \\
\hline Aina Gionés Valls & Bauen & Estudiante & - & - & - & - & - & - & - \\
\hline Daniel Gil Solés & Bauen & Bibliotecario & $x$ & $x$ & $x$ & $x$ & - & $x$ & \\
\hline Álvaro Roldan & Bibliometria & $\begin{array}{l}\text { Administración } \\
\text { pública }\end{array}$ & $x$ & - & $x$ & $x$ & - & - & - \\
\hline $\begin{array}{l}\text { Javier Leiva } \\
\text { Aguilera }\end{array}$ & $\begin{array}{l}\text { Catorze/ } \\
\text { Véase además }\end{array}$ & $\begin{array}{l}\text { Profesor } \\
\text { universitario }\end{array}$ & $x$ & - & $x$ & $x$ & $x$ & - & - \\
\hline $\begin{array}{l}\text { Catuxa Seoane } \\
\text { García }\end{array}$ & Deakialli & Bibliotecaria & $x$ & - & - & $x$ & - & $x$ & $x$ \\
\hline $\begin{array}{l}\text { Vanesa Barrero } \\
\text { Robledo }\end{array}$ & Deakialli & Documentalista & - & - & - & - & - & - & - \\
\hline $\begin{array}{l}\text { Álvaro Cabezas } \\
\text { Clavijo }\end{array}$ & Doc., bib. e inf. & $\begin{array}{l}\text { Becario } \\
\text { investigación }\end{array}$ & $x$ & - & $x$ & $x$ & - & - & - \\
\hline Marcos Ros Martín & Doc. enredado & Documentalista & - & - & $x$ & $x$ & $x$ & $x$ & $x$ \\
\hline Maria Elena Mateo & Doc. enredado & Documentalista & - & - & $x$ & - & - & - & - \\
\hline Núria Gallart & Gamoia & Bibliotecaria & $x$ & $x$ & $x$ & - & - & $x$ & $x$ \\
\hline $\begin{array}{l}\text { Ricardo Fornas } \\
\text { Carrasco }\end{array}$ & $\begin{array}{l}\text { Métodos de } \\
\text { busca }\end{array}$ & Consultor & - & - & $x$ & - & $x$ & $x$ & \\
\hline $\begin{array}{l}\text { Fo Javier Martínez } \\
\text { Méndez }\end{array}$ & RI en la web & $\begin{array}{l}\text { Profesor } \\
\text { universitario }\end{array}$ & - & - & $x$ & - & - & $x$ & - \\
\hline $\begin{array}{l}\text { Luis Rodríguez } \\
\text { Yunta }\end{array}$ & Sedic & Documentalista & $x$ & $x$ & $x$ & - & $x$ & $x$ & - \\
\hline Elisa Prieto Castro & Sedic & Bibliotecaria & $x$ & - & $x$ & - & $x$ & - & - \\
\hline $\begin{array}{l}\text { Nuria Escudero } \\
\text { Galán }\end{array}$ & Sedic & $\begin{array}{l}\text { Espec. en } \\
\text { informac. }\end{array}$ & - & - & $x$ & - & - & $x$ & - \\
\hline $\begin{array}{l}\text { María Jesús } \\
\text { Butera Fajardo }\end{array}$ & Sedic & Documentalista & $x$ & - & $x$ & $x$ & - & $x$ & - \\
\hline $\begin{array}{l}\text { Virginia Becerril } \\
\text { González }\end{array}$ & Sedic & Documentalista & $x$ & - & $x$ & - & - & - & - \\
\hline $\begin{array}{l}\text { Julio Igualador } \\
\text { Osoro }\end{array}$ & Sedic & Documentalista & - & - & $x$ & - & - & - & - \\
\hline $\begin{array}{l}\text { Juan Carlos García } \\
\text { Gómez }\end{array}$ & Usalo & Documentalista & $x$ & $x$ & $x$ & - & $x$ & $x$ & $x$ \\
\hline $\begin{array}{l}\text { Tomás Saorín } \\
\text { Pérez }\end{array}$ & Usalo & $\begin{array}{l}\text { Administración } \\
\text { pública }\end{array}$ & $x$ & $x$ & $x$ & $x$ & - & $x$ & - \\
\hline $\begin{array}{l}\text { Yusef Hassan } \\
\text { Montero }\end{array}$ & Véase además & Investigador & $x$ & - & $x$ & - & $x$ & $x$ & $x$ \\
\hline $\begin{array}{l}\text { Paola Andrea } \\
\text { Ramírez }\end{array}$ & Véase además & $\begin{array}{l}\text { Profesora } \\
\text { universitaria }\end{array}$ & - & - & - & $x$ & $x$ & - & - \\
\hline Fernando Siles & Véase además & Documentalista & - & - & - & - & - & - & - \\
\hline
\end{tabular}

Tabla 5. Autores de los blogs de ByD: profesión y medios de comunicación empleados

derecho. Todos ellos, dedicados a diversos ámbitos de la $\mathrm{ByD}$, son los que más producen y los que generan un mayor debate en la bibliogosfera y pueden ser considerados como el núcleo de la disciplina.

En el cuadrante superior izquierdo se localizan los blogs que publican pocos posts pero que reciben un número significativo de comentarios. Encontramos los muy especializados que tratan temáticas documentales muy específicas, como la bibliometría y la usabilidad. En el cuadrante inferior derecho están dos con gran número de posts publicados pero con poca capacidad para atraer comentarios. Por último, en el cuadrante inferior izquierdo encontramos los peor posicionados, aquellos que producen poco y además reciben pocos comentarios.

"Dentro del grupo de 12 blogs más relevantes hay una tendencia clara hacia la caída en la redacción de posts" 


\begin{tabular}{|l|r|r|r|r|r|r|r|r|}
\hline \multicolumn{1}{|c|}{ Blog } & P & PxD & Pr & PrxP & C & CxP & PSC & \%PSC \\
\hline Bauen & 250 & 0,5 & 71.898 & 288 & 170 & 0,7 & 181 & 72 \\
\hline Bibliometría & 80 & 0,2 & 20.722 & 259 & 297 & 3,7 & 29 & 36 \\
\hline Catorze & 219 & 0,5 & 24.540 & 112 & 1.133 & 5,2 & 66 & 30 \\
\hline Deakialli & 342 & 0,7 & 59.208 & 173 & 1.107 & 3,2 & 126 & 37 \\
\hline Documentacion, bibl. e inf. & 146 & 0,3 & 32.574 & 223 & 726 & 5,0 & 26 & 18 \\
\hline Documentalista enredado & 192 & 0,4 & 115.563 & 602 & 659 & 3,4 & 38 & 20 \\
\hline Gamoia & 88 & 0,2 & 23.959 & 272 & 243 & 2,8 & 34 & 39 \\
\hline Métodos de busca & 21 & 0,0 & 2.532 & 121 & 2 & 0,1 & 20 & 95 \\
\hline RI en la Web & 182 & 0,4 & 58.360 & 321 & 127 & 0,7 & 126 & 69 \\
\hline Sedic & 116 & 0,2 & 27.034 & 233 & 118 & 1,0 & 71 & 61 \\
\hline Úsalo & 74 & 0,2 & 36.581 & 494 & 320 & 4,3 & 8 & 11 \\
\hline Véase además & 260 & 0,5 & 25.365 & 98 & 827 & 3,2 & 97 & 37 \\
\hline
\end{tabular}

Tabla 6. Indicadores generales para los blogs de ByD: posts, palabras y comentarios

\section{Discusión y conclusiones}

A raíz de los resultados y retomando como herramienta de análisis los comentarios efectuados en el post ¿Se han estancado los blogs documentales? (véase cuadro de texto adjunto) se puede concluir básicamente lo siguiente:

Existe un grupo de 12 blogs que son los más relevantes de la bibliogosfera nacional si consideramos el indicador "Autoridad". Dentro de este grupo hay una tendencia clara hacia la caída en la redacción de posts. Este descenso ha provocado también que decrezca el número bruto de comentarios por lo que los debates y la actividad de los lectores se ha visto reducida al no tener contenidos para ello, la ratio comentarios/post no indica en ningún momento que se produzca una menor participación. Igual parece ocurrir con la longitud, aunque se publique menos, las entradas mantienen una extensión similar a lo largo del período. Por tanto se constata una menor actividad en la generación de contenidos pero no en la participación.

- Esta tendencia decreciente de la producción es síntoma evidente de un desgaste y de la adquisición de una posición acomodada dentro de la comunidad profesional de blogs documentales. La creciente especialización de algunos de ellos, la falta de tiempo por tenerse que dedicar a un puesto de trabajo (al que muchas veces se ha accedido gracias a la excelente plataforma profesional que significa mantener un blog de calidad) o la búsqueda de canales más apegados a la comunicación científica tradicional (artículos, congresos, etc.) son algunas de las causas aducidas por los propios bloggers que, en general, reconocen la bajada de la actividad.

- El análisis de los comentarios ha ilustrado diversos aspectos sobre la participación. Nos encontra- mos ante una comunidad bastante reducida y cerrada compuesta activamente por unas decenas de personas. Pese a ser la blogosfera un espacio abierto a cualquier lector, sólo se consigue un $43 \%$ de comentarios externos, es decir de personas no vinculadas a ningún blog documental. Esta situación es característica de un grupo endogámico con fuertes lazos internos que se reparte la mayor parte de los comentarios entre ellos mismos. Las cifras globales de participación y el perfil de los participantes en cierta medida revelan el escaso interés y el desconocimiento que la comunidad de ByD le presta a los blogs como medio de comunicación para el intercambio de experiencias profesionales y científicas. Este fenómeno queda reflejado también en el número de posts sin comentarios que se situó en el $42 \%$ del total.

\section{"Es una comunidad bastante reducida y cerrada compuesta activamente por unas decenas de personas"}

- Los comentarios tienden a concentrarse en posts poco relevantes que temáticamente guardan una escasa o nula relación con la ByD. En este sentido no se puede afirmar que el número de comentarios se corresponda con la calidad de los contenidos o la influencia de los autores en la disciplina; contrariamente: los temas populares consiguen atraer más comentaristas que poco pueden aportar. Se sitúan también entre los posts más comentados aquellos directamente relacionados con los blogs, un tema recurrente sobre el que, evidentemente, 


\section{Comentarios destacados realizados sobre el post ¿Se han estancado los blogs documentales? publicado en el blog Documentación, biblioteconomía e información.}

- "Me parece muy interesante este mini estudio y simboliza perfectamente una cosa: desgaste" (Marcos Ros, El documentalista enredado).

- "Creo que la reducción en el número de comentarios obedece a dos razones. Por una parte, el aumento del uso de herramientas de sindicación de contenidos hace que muchos lectores lean los posts sin acceder al blog y que, por tanto, no lleguen a leer los comentarios (a los que rara vez se suscribe alguien). Por otra parte, a mayor especialización de los blogs menor número de comentarios" (Álvaro Roldán, Bibliometria.com).

- "Estaría bien hacer un análisis de quién comenta, dónde y con qué frecuencia. Si los debates en una comunidad de blogs se nutren de comentarios procedentes fundamentalmente de miembros de esa misma comunidad, y la actividad de ésta decae en lo que a producción de contenidos se refiere, parece lógico que también se reduzcan sus comentarios. Al fin y al cabo, la actividad bloguera no se limita (generalmente) a escribir en el blog propio, sino que va todo unido (leer-comentar-escribir)". (Paco Fernández, Archivista).

- "La mayoría de autores citados han/habéis "crecido" profesionalmente lo cual os ha abierto las puertas a otras formas de comunicación. Lo mismo ha sucedido con determinados "bloggers" americanos, que han dejado de publicar posts sustanciosos en la misma medida que han aumentado sus artículos en revistas, libros, cursillos, etc.". (Dídac Margaix, Dospuntocero).

- "Echo en falta sobre todo los blogs de investigadores y docentes, que sin duda alguna podrían aprovechar los blogs como medio de comunicación; sin embargo, tengo la sensación de que siguen demasiado enfrascados en los canales tradicionales de comunicación: jornadas, congresos y artículos en publicaciones especializadas". (María-José Butera, Sedicblog).

- "Ahora hay miles de novedades cada día, por suerte la mayoría hemos evolucionado mucho profesionalmente y estamos muy ocupados (eso aborta muchos posts), el formato ha madurado y nosotros con él, hemos ido cambiando de gustos y seguramente muchos visitamos blogs de temas que antes no visitábamos, hay más gente posteando (aunque pudiera haber aún más, claro), etc. No sé, yo no creo en absoluto que los blogs sobre este sector gocen de mala salud". (Javier Leiva, Catorze).

- "Si se hiciera una clasificación de blogs de temática bibliodocumental debería haber numerosos huecos vacíos o al menos blogs duplicados y no los hay. Faltan temas por cubrir, yo creo. Hemos crecido muy poco en número". (Vanesa Barrero, Deakialli).

http://www.lacoctelera.com/documentacion/ post/2007/05/08/ase-han-estancado-blogsdocumentales- todo blogger tiene algo que decir. Esta concentración temática es síntoma de la escasa cobertura de la ByD en general. La discusión de la blogosfera poco tiene que ver con los frentes de investigación en ByD o con las materias clásicas de la diplomatura y la licenciatura.

- No hay un perfil que defina claramente al conjunto de bloggers; todos ellos se dedican a diferentes ramas profesionales de la $\mathrm{ByD}$, pero no existen unas características comunes dominantes; el rango profesional abarca desde estudiantes hasta profesores pasando por investigadores y personal de la administración. Estos autores aunque emplean los blogs como medio de
"La discusión de la blogosfera tiene poco que ver con los frentes de investigación en ByD o con las materias clásicas de la diplomatura y la licenciatura"

comunicación preferente no se limitan a los mismos. Los medios tradicionales también son utilizados, sobre todo las revistas científicas y las comunicaciones a congresos. La difusión en estos canales suele ser de 
ámbito nacional e incluso regional. En ocasiones colaboran además en otros blogs y medios digitales y algunos transmiten sus experiencias profesionales a través de cursos.

- De los 12 weblogs estudiados resalta un grupo de cuatro que pueden ser considerados los más importantes, en cuanto a producción y comentarios por post. Estos son Deakialli, Véase además, Catorze y Documentalista enredado. Son, asimismo, los que más enlaces reciben, y sus autores poseen una experiencia acumulada de entre 3-4 años en la redacción de sus blogs.

\section{Bibliografía}

Bar-Ilan, J. "Information hub blogs". En: Journal of information science, 2005, v. 31, n. 4, pp. 297-307.

Becerril, V.; Butera, M. J.; Escudero, N.; Igualador, J.; Prieto, E.; Rodríguez-Yunta, L. "Experiencia con el blog colectivo de Sedic". En: El profesional de la información, 2007, v. 16, n. 2, pp. 134-137.

Cabezas, Á. ¿Se han estancado los blogs documentales?. En: Documentación, biblioteconomía e información. Consultado en: 10-07-07.

http://www.lacoctelera.com/documentacion/post/2007/05/08/ase-han-estancado-blogs-documentales-

Carroll, D. Technorati authority and rank. Consultado en: 08-07-07. http://technorati.com/weblog/2007/05/354.html

Crawford, W. "Investigating the biblioblogosphere". En: Cites \& insights: crawford at large, 2005, v. 5, n. 10, pp. 2-13.

Ferrada, M. Biblogsfera, comunidad de bitácoras cuya temática versa total o parcialmente sobre biblioteconomía y documentación. Consultado en: 08-07-07.

http://eprints.rclis.org/archive/00007179/01/ferrada_biblogsfera.pdf

Flores, B.; Legerén E. "El fenómeno weblog como nuevo medio de comunicación: su incidencia en el campo de la biblioteconomía y la documentación”. En: I Encuentro de estudiantes para la organización del conocimiento, $7^{\circ}$ congreso ISKO-España, 2005.

Franganillo, J.; Catalán, M. A. "Bitácoras y sindicación de contenidos: dos herramientas para difundir información". En: BiD: textos universitaris de biblioteconomia i documentació, 2005, diciembre, n. 15

Jiménez-Contreras, E.; Delgado-López-Cózar, E.; Ruiz-Pérez, R. "Producción española en biblioteconomía y documentación con visibilidad internacional a través del Web of Science (1995-2004)". En: El profesional de la información, 2006, v. 15, n. 5, pp. 373-383.

Jiménez-Hidalgo, S.; Salvador-Bruna, J. "Evaluación formal de blogs con contenidos académicos y de investigación en el área de documentación”. En: El profesional de la información, 2007, v. 16, n. 2, pp. 114-22.

Leiva, J. "Blogs: una herramienta de difusión para profesionales de la información". En: TK, 2006, n. 18.

Lledó, M. El profesional de la información ante los weblogs. Consultado en: 08-07-07.

http://eprints.rclis.org/archive/ 00000498/01/mlledo_weblogs.pdf

López-Carreño, R.; Martínez-Méndez, F. J. "Revisión de los servicios de internet: su aprovechamiento en unidades de información y documentación”. En: Revista española de documentación científica, 2005, v. 28, n. 4, pp. 585-609.

López, F.; Penadés, H. "365 días de libros: blog para la promoción de la lectura". En: El profesional de la información, 2007, v. 16, n. 2, pp. 131133

Merlo-Vega, J. A.; Sorli-Rojo, Á. "Weblogs: un recurso para los profesionales de la información”. En: Revista española de documentación científica, 2003, v. 26, n. 2, pp. 227-236.

Rojas, O.; Alonso, J.; Antúnez, J. L.; Orihuela, J. L.; Varela, J. Blogs. La conversación en internet que está revolucionando medios, empresas y ciudadanos. Madrid: ESIC, 2005. Isbn 84-7356-427-8.

Ros, M.; Mateo, M. E. Análisis de la biblioblogosfera hispana (4). Estudio cibermétrico: quién y cómo. En: El documentalista enredado. Consultado en: 10-07-07.

http://www.documentalistaenredado.net $/ ? p=285$

Ros, M.; Mateo, M. E. Análisis de la biblioblogosfera hispana (7), cronología. En: El documentalista enredado. Consultado en: 10-07-07. http://www.documentalistaenredado.net/?p=288

Sedic blog equipo de redacción. "Blogs de biblioteconomía y documentación en España". En: Revista española de documentación científica, 2006, v. 29 , n. 4 , pp. $603-27$

Daniel Torres-Salinas, Centro de Investigación Médica Aplicada. Universidad de Navarra torressalinas@gmail.com

Álvaro Cabezas-Clavijo, Departamento de Biblioteconomía y Documentación. Universidad de Granada. acabezasclavijo@gmail.com

Emilio Delgado-López-Cózar, Departamento de Biblioteconomía y Documentación. Universidad de Granada.

edelgado@ugr.es

\section{Suscripción EPI sólo online}

Pensando sobre todo en los posibles suscriptores latinoamericanos, ya no es obligatorio pagar la suscripción impresa de EPI para acceder a la online.

EPI se ofrece a instituciones en suscripción "sólo online" a un precio considerablemente más reducido (85 euros/año), puesto que en esta modalidad no hay que cubrir los gastos de imprenta ni de correo postal. 